\title{
A New Hypothesis for Dystonia
}

In this issue of the Annals, Goto and colleagues present a new hypothesis concerning the pathophysiology of dystonia. ${ }^{1}$ A measure of our limited understanding of dystonias is the fact that although dystonia is a brain disorder, the most effective treatment is botulinum toxin chemodenervation at the neuromuscular junction. Ignorance of the mechanisms of dystonia is an obstacle to developing mechanism-based therapies and to evaluating putative animal models. Accumulated data indicate that dystonias result from basal ganglia dysfunction, a conclusion supported by lesion data, neuroimaging results, and most recently by electrophysiological data from dystonic patients. An intriguing idea is that primary basal ganglia dysfunction causes abnormalities of sensorimotor integration at the level of the neocortex. ${ }^{2}$

Goto and colleagues use a sophisticated version of traditional clinicopathological correlation to investigate striatal changes in X-linked dystonia parkinsonism (XPD; DYT3; Lubag), a disorder endemic on the island of Panay in the Philippines. In XPD, men develop marked, usually generalized, dystonia followed by parkinsonism in the later years of life. This experiment of nature allows comparison of striatal changes in the dystonic and parkinsonian phases of the disease. Striatal neurons consist of two broad categories, interneurons whose axonal arborizations are restricted to the striatum itself, and GABAergic projection neurons whose primary axons project to the pallidum or the substantia nigra. Projection neurons comprise the great majority of striatal neurons and can be subdivided further on the basis of their projection targets, neuropeptide expression, and expression of other markers, notably some calcium binding proteins. A basic distinction is between striosomal projection neurons and matrix projection neurons. Striosomal projection neurons project mainly to dopaminergic substantia nigra pars compacta neurons, whereas the matrix contains relatively segregated populations of neurons projecting to the different components of the globus pallidus or the substantia nigra pars reticulata. Differential loss or dysfunction of matrix projection neurons is correlated with other movement disorders. ${ }^{3-5}$ Preferential, early loss of matrix neurons projecting to the lateral segment of the globus pallidus is correlated with expression of choreoathetosis in Huntington's disease. In Parkinson's disease, loss of striatal dopaminergic innervation alters the balance of matrix projection neuron subpopulation activities in a way that results in altered basal ganglia output to the thalamus.

Using immunohistochemistry to identify various populations of striatal projection neurons, Goto and colleagues show convincingly that dystonic XPD (XPD-D) is marked by differential loss of striatal projection neuron subpopulations. They show very nicely that striosomal projection neurons are lost. Their data indicate partial loss of matrix projection neurons as well in XPD-D with what seems to be approximately equivalent loss of matrix neurons projecting to either segment of the globus pallidus or the substantia nigra pars reticulata. In the more advanced XPD-P cases, virtually all striatal projection neurons appear to be lost. This leads to the suggestion that dystonia results from preferential loss of striosomal projection neurons, perhaps altering the behavior of dopaminergic nigrostriatal neurons in a way that causes dystonia. This hypothesis has several attractions. It represents a further and novel articulation of popular models of basal ganglia dysfunction based on degeneration or dysfunction of matrix projection neurons. These models had difficulty explaining dystonia. ${ }^{6}$ In addition, Goto and colleagues' suggestion that alteration of dopaminergic neuron activity is involved in the pathophysiology of dystonia also makes sense. Some known causes of dystonia, such as dopa-responsive dystonia (DRD; DYT5; Segawa's syndrome) or dystonic phenomena in fluctuating Parkinson's patients, definitely involve dopaminergic dysfunction.

These data admit another interpretation. Connectional and some physiological data suggest the existence of fine-grained matrix compartments in an organization that is based more on patterns of corticostriate innervation than output connections, so-called matrisomes. ${ }^{7-10}$ It is possible that Goto and colleagues have identified both relatively preferential loss of striosomal projection neurons and some subpopulations of matrisomal neurons. Dystonia could result from early, preferential loss of specific matrisome subpopulations or from combined loss of striosomal projection neurons and some matrisome subpopulations. The question of which interpretation of these data is correct is much less important than the fact that these hypotheses suggest fruitful and doable experiments. Striatal specimens from subjects with early mild XPD or other forms of generalized dystonia should be examined for evidence of striosomal and matrisomal projection neuron loss. Further work along these lines could markedly advance our understanding of dystonia. The results of Goto and colleagues underscore the continuing importance that understanding the basic anatomy and physiology of the basal ganglia has for exploring movement disorders. 
Roger L. Albin, $M D$

Geriatrics Research, Education, and Clinical Center, Ann Arbor VAMC

Department of Neurology, University of Michigan

Ann Arbor, MI

\section{References}

1. Goto S, Lee LV, Dantes MB, et al. Functional anatomy of the basal ganglia in X-linked recessive dystonia-parkinsonism. Ann Neurol 2005;58:7-17.

2. Hallett M. Dystonia: abnormal movements result from loss of inhibition. Adv Neurol 2004;94:1-9.

3. Albin RL, Young AB, Penney JB. The functional anatomy of basal ganglia disorders. Trends Neurosci 1989;12:366-375.

4. DeLong MR. Primate models of movement disorders of basal ganglia origin. Trends Neurosci 1990;13:281-285.
5. Crossman AR. Primate models of dyskinesia: the experimental approach to the study of basal ganglia-related involuntary movement disorders. Neuroscience 1987;21:1-40.

6. Albin, RL, Young AB, Penney JB. The functional anatomy of disorders of the basal ganglia. Trends Neurosci 1995;18:63-64.

7. Flaherty AW, Graybiel AM. Two input systems for body representations in the primate striatal matrix: experimental evidence in the squirrel monkey. J Neurosci 1993;13:1120-1137.

8. Flaherty AW, Graybiel AM. Output architecture of the primate putamen. J Neurosci 1993;13:3222-3237.

9. Kincaid AE, Wilson CJ. Corticostriatal innervation of the patch and matrix in the rat neostriatum. J Comp Neurol 1996;374: $578-592$.

10. Zheng T, Wilson CJ. Corticostriatal combinatorics: the implications of corticostriatal axonal arborizations. J Neurophysiol 2002;87:1007-1017.

DOI: $10.1002 /$ ana.20520 